\title{
Solid parotid tumors: an individual and integrative analysis of various ultrasonographic criteria. A prospective and observational study.
}

\author{
Alexandru Florin Badea ${ }^{1}$, Simion Bran¹, AttilaTamas-Szora ${ }^{2}$, Alexandru Floareş², \\ Radu Badea², Grigore Baciut ${ }^{1}$
}

${ }^{1}$ Department of Maxillofacial Surgery, ${ }^{2}$ Department of Ultrasonography, “O. Fodor" Institute of Gastroenterology and Hepatology, "Iuliu Hațieganu" University of Medicine and Pharmacy, ${ }^{3}$ Artificial Intelligence Department of SAIA and OncoPredict, Cluj Napoca, Romania

\begin{abstract}
Objectives: The purpose of the study is to identify and validate ultrasound criteria for parotid tumors evaluation, as well as to elaborate a multimodal, multi-criteria and integrative ultrasound approach for allowing tumor discrimination in a noninvasive manner. Material and method: Twenty patients with solid parotid tumors (12 benign, 8 malignant) were examined by ultrasound: real-time "grey scale" ultrasound, Doppler ultrasound, elastography, harmonic ultrasound imaging with i.v. contrast (CEUS). The study focused on tumor morphology and circulation. The analysis of the results was observational, enhanced by statistical methods and artificial intelligence (decision trees). Results: All malignant tumors showed increased hypoechogenicity, tumoral cervical adenopathies, increased stiffness and ,in block" mobility with the parotid gland upon palpation with the transducer, uneven distribution of the contrast material during the arterial phase (8/8). To varying degrees, they showed imprecise delineation (7/8), structural heterogeneity (6/8) and disorganized flow pattern (6/8). All cases of benign tumors showed heterogeneous echostructure, clear delineation and no capsule (12). They also showed moderate hypoechogenicity (9/12), no cervical lymph nodes (11/12) and variable rigidity (increased 6/12; low 3/12). A selection and ranking of relevant ultrasound parameters was also made. Some of them were included in a transparent and easy-to-use decision tree model with $100 \%$ data accuracy. Conclusions: The characterization and discrimination of solid parotid tumors require a multimodal and multicriteria approach. Ultrasound criteria can be divided into criteria of certainty and criteria of diagnosis probability. CEUS examination of parotid tumors did not reveal significant differences between benign and malignant circulatory bed. Decision trees discovered by artificial intelligence from the data may represent intelligent diagnosis support systems with very high accuracy, up to $100 \%$.
\end{abstract}

Keywords: parotid tumor; ultrasound; elastography; contrast agent; harmonic.

\section{Introduction}

Salivary gland tumors have a relatively low incidence (1-5 cases $/ 100,000$ inhabitants), representing $2-6 \%$ of head and neck tumors, $85 \%$ of these tumors involving

Received 03.09.2013 Accepted 08.10.2013

Med Ultrason

2013, Vol. 15, No 4, 289-298

Corresponding author: Radu Badea,

Department of Ultrasonography,

$3^{\text {rd }}$ Medical Clinic, Gastroenterology and

Hepatology Institute,

"Iuliu Hațieganu" University of Medicine and Pharmacy,

19-21 Croitorilor str

400494 Cluj Napoca, Romania

Email: rbadea@umfcluj.ro the parotid gland [1]. The detection and characterization of parotid masses is important considering the complex anatomical relationships with the vascular and nervous structures of the region and the morbidity associated with extensive surgery situations that can lead to disability [2].

The grey scale ultrasound (US) imaging is considered the first procedure to be performed immediately after the clinical examination in order to assess the cervical masses. The advantages of this method are: high availability, relatively low cost and a good enough accuracy that consolidates the clinical diagnosis and directs further investigations. US is non-radiating and therefore highly recommended for children or pregnant women. A specific element of this procedure is the visualization of the dynamic organs (heart, digestive tract, fetal mobility) and 
of the motility of different biological structures (blood, urinary stream) at the time of the event ("real time" imaging) which gives to it a pronounced clinical character [3].

US cannot substitute other imaging procedures such as Computer Tomography (CT) or magnetic resonance imaging (MRI) because of its well-known limitations: different basic design principles, dependence on the operator and equipment, lack of standardization of sections and hence the variability of information from one examination to another, ultrasound attenuation during propagation and difficult or impossible access to the deep structures of the neck. Therefore the method is complementary for the pre-treatment investigation battery, being closer to the clinical examination than to imaging $[4,5]$.

Currently used US procedures are "grey scale" exploration and Doppler ultrasound. In the recent years, technological developments have allowed the introduction into practice of new evaluation modules that provide additional information: elastography, available in different versions (qualitative colour coded and quantitative), and contrast enhanced ultrasound (CEUS) [6,7]. Each of these new procedures brings specific information that must be integrated with the ultrasound examinations and contributes to the non-invasive characterization, without biopsy, of the nature of tumoral masses. Selecting and ranking US parameters with diagnosis value is an important task that can be realized by using statistical methods that test their association with the diagnosis. In addition, the use of artificial intelligence in a "data mining" approach allows the development of transparent models, which are easy to interpret and use, and have high accuracy.

The aims of the study are to a) identify the sonographic features, (morphological and vascular) of solid parotid tumors; b) develop a multimodal ultrasound model which intends to be integrative, accurate and useful for the positive and differential diagnosis of benign and malignant parotid solid tumors; c) select and rank ultrasound parameters, as well as to develop an intelligent diagnosis support system in the form of a decision tree.

\section{Material and method}

The study (prospective and linear) was conducted on 24 patients and then downsized (two cases presented cysts and the other two diffuse parotid inflammation) to 20 subjects ( 5 women, 15 men, age between $40-72$ years). All the subjects presented unilateral parotid masses that were clinically detected. The patients were identified and enrolled in the Ambulatory Department of the Maxillofacial Surgery Clinic, Cluj-Napoca between April and December 2012.
The inclusion criteria were: cervical mass with/without clinical expression, association with the parotid gland demonstrated by ultrasound and/or CT/MRI and final diagnosis established after surgery and antomopathology examination. The exclusion criteria were: cervical masses whose organ association or nature could not be demonstrated, cervical lymph-node syndromes, intraglandular adenopathies, acute or chronic parotitis, parotid fluid collections, or abscesses.

All patients were informed about the study and gave their written consent. The study was approved by the Ethics Committee of the University.

All the patients in the study group underwent physical and US examination.

The US examination was performed using multiple devices: General Electric GE 7, GE 8, GE 9, iU22 Phillips, Siemens S 2000. In all cases the exploration procedure was standardized: the patient was placed supine, facing upwards.

The pathological region and the normal parotid region, considered as witness, were comparatively examined. In all cases, a high frequency transducer 7-11 MHz was used. The US criteria used for the characterization of solid parotid tumors and the codification modality used to process the information are detailed in Table I.

The "grey-scale", bi-dimensional examination (2D) was the procedure by which the tumor mass was detected and the clinical diagnosis of the tumor was strengthened. The elastographic examination was performed using a colour coded procedure, either "tactile elastography " either Acoustic Radiation Force Impulse (ARFI). For the Doppler examination the equipment was set for velocities below $15 \mathrm{~cm} / \mathrm{sec}$, arterial resistance was assessed as low or high based on the ratio of systolic and diastolic velocities and velocities were detected by placing the Doppler sample in the centre of the vessel with a correction angle of less than 60 degrees. The CEUS examination used a standardized procedure [8] consisting in the administration of $1.6 \mathrm{cc}$ of SonoVue, extemporaneously prepared, injected into the cubital vein, followed by a bolus injection of $10 \mathrm{cc}$ of saline solution. A dual examination, grey scale and harmonic, with the ultrasound information displayed simultaneously on the US monitor was performed in all situations. The exploration was carried out continuously from the time of injection for about 120 seconds. A mechanical index of 0.10 was used and the focus was placed below the tumor which was considered the region of interest (ROI).

In all the cases a confrontation with the histology results obtained by analysing the surgery specimens was performed. 
Med Ultrason 2013; 15(4): 289-298 291

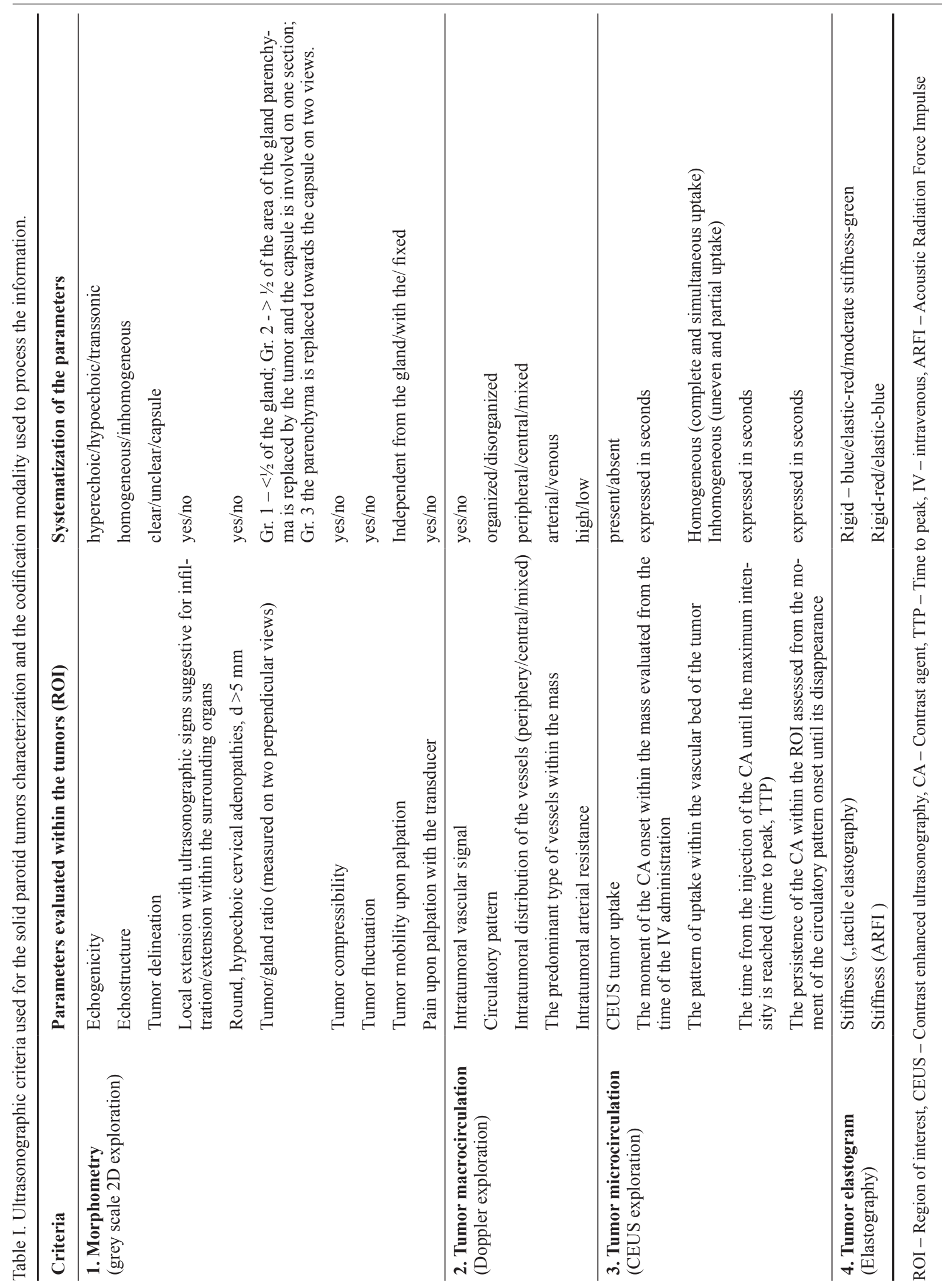


The data analysis was observational and was processed through statistical and artificial intelligence methods, in a data-mining approach. The statistical methods aimed at 1) the elimination of the ultrasound parameters which, in the context of the present study, are uninformative and 2) the selection and ranking of the relevant variables. The hierarchy was developed on the basis of significance, defined as $(1-p)$, where $p$ represents the p-value of the statistical test of association between the ultrasound parameter and the diagnosis. The Pearson Chi-square statistical test was used. The Mann Whitney test was used for mean comparison (statistical significance was considered as being $\mathrm{p}<0.05$ ). The artificial intelligence methods of CART decision trees (Classification And Regression Trees) were also used, as they provide models of high accuracy that are easy to use and interpret. The study aimed at defining the decision trees characterized by the highest accuracy, using the fewest and most robust ultrasound parameters

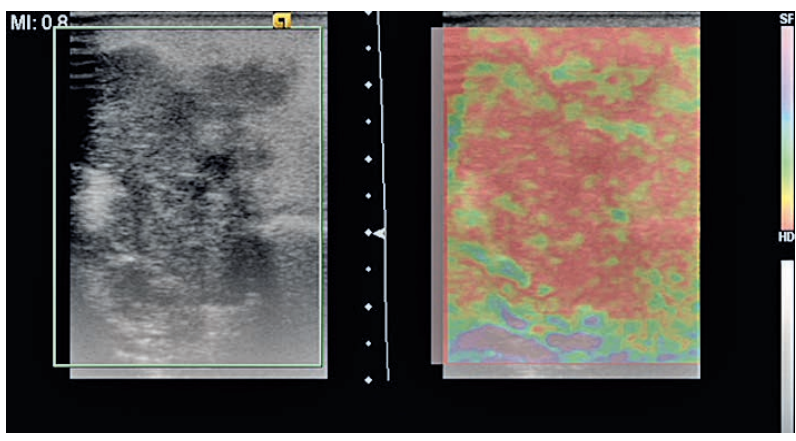

Fig 1. Parotid carcinoma. ARFI technique elastography. The tumor is irregularly and unclearly delineated. There is an overall increased stiffness of the tumor (red color).

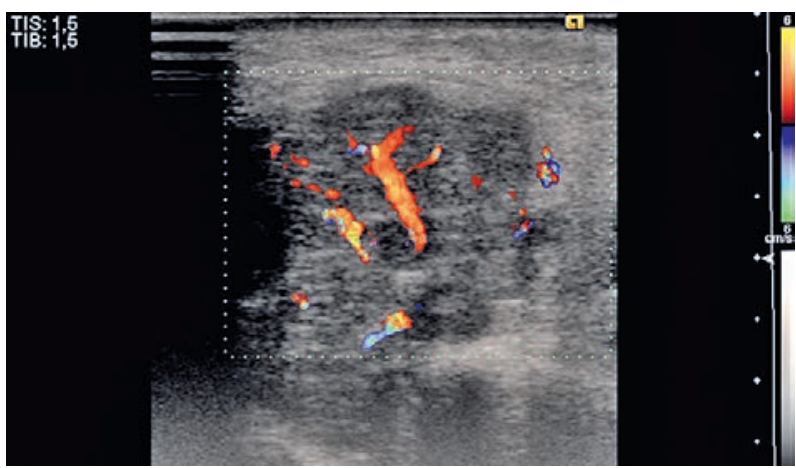

Fig 2. Parotid carcinoma. Color coded Doppler ultrasonography (CFM). The circulatory bed contains disorganized vessels, with a variable lumen caliber, with discontinuities caused by the tumor parenchyma. possible (for an introductory description of the methods see [9]).

\section{Results}

In the study group were identified 8 malignant (6 parotid carcinomas, 2 metastases - one was from a melanoma and the other from a primary carcinoma of the scalp) and 12 benign tumors (all pleomorphic adenomas). The comparative findings in malignant and benign solid parotid tumors obtained after US examination are detailed in table II and relevant images are presented in figures $1-8$.

No significant statistical differences were noted between the groups when comparing the means of the CEUS parameters ( $\mathrm{p}>0.05$ ) (fig 9-11).

All the parameters investigated were ranked according to their importance, and the irrelevant parameters were eliminated (the texture characterization in reference

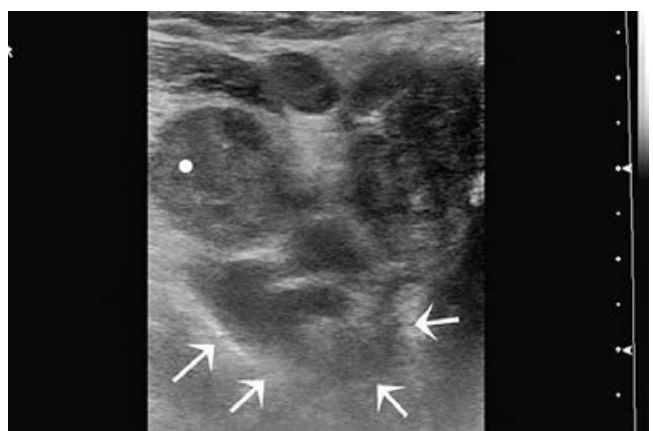

Fig 3. Parotid carcinoma. "Grey scale" ultrasound. The tumor inhomogeneous and highly hypoechoic. The capsule of the tumor has discontinuities and there is tumor invasion in the surrounding cervical structures (arrows). There are round, inhomogeneous adenopathies with tumoral features (white dot).

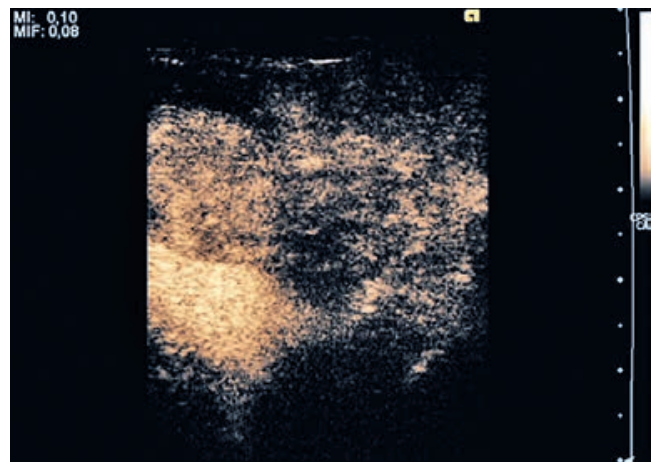

Fig 4. Parotid carcinoma. CEUS exam. Inhomogeneous enhancement pattern (arterial phase). 
Med Ultrason 2013; 15(4): 289-298 293

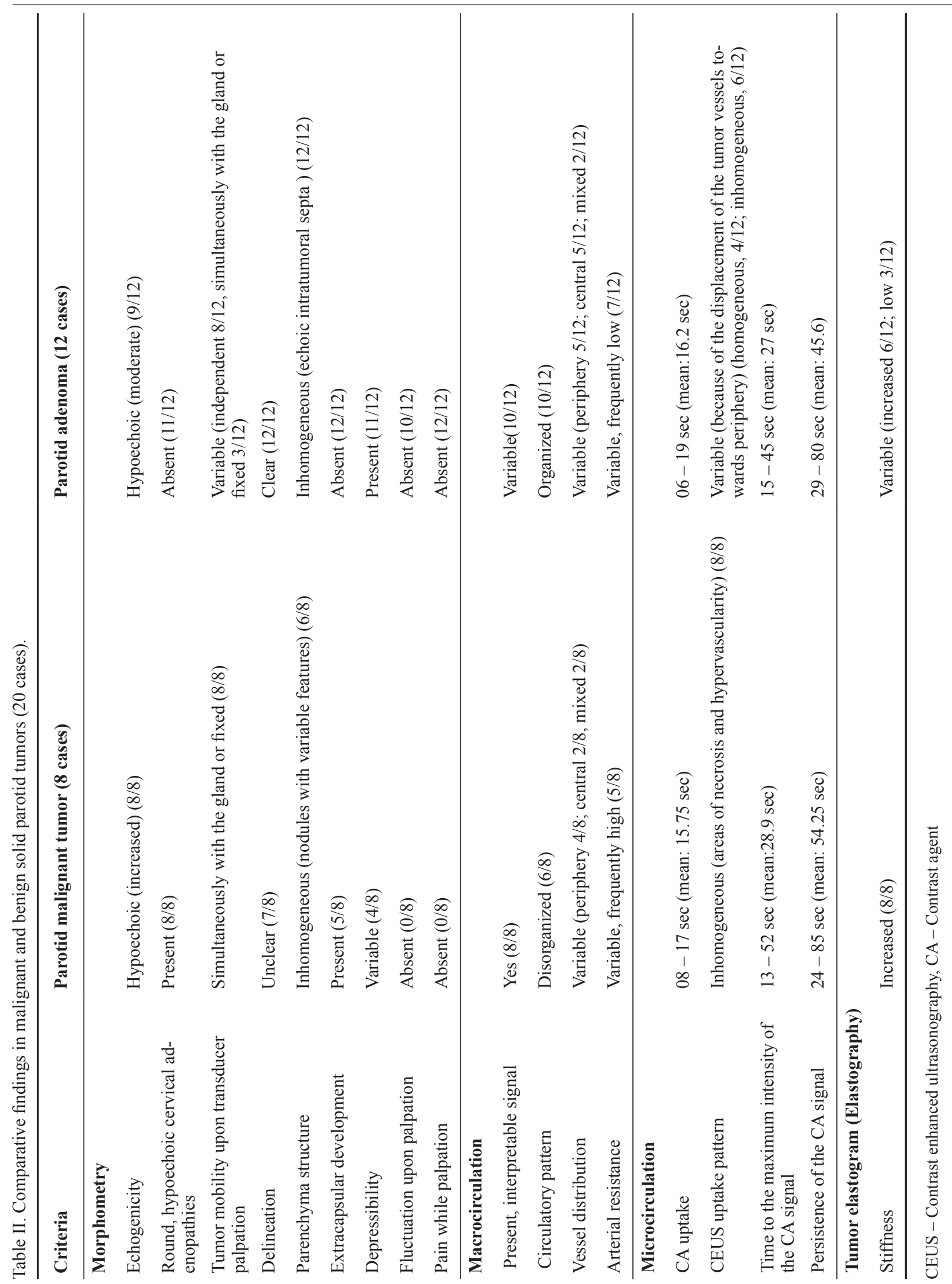




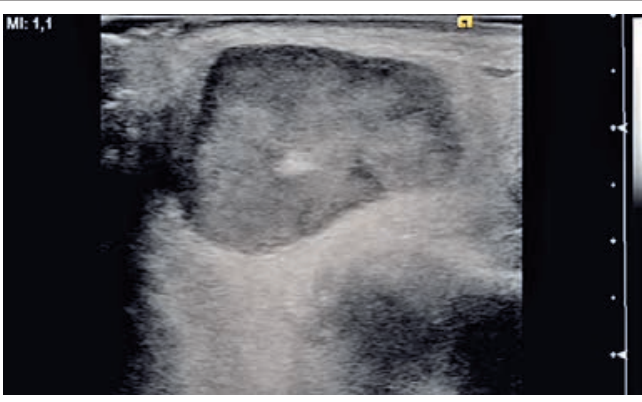

Fig 5. Pleomorphic parotid adenoma. "Grey scale" ultrasound. The structure is mildly inhomogeneous (generated by a restructured parenchyma and fibrous, echoic septa). The tumor is clearly delineated, without any discontinuities.

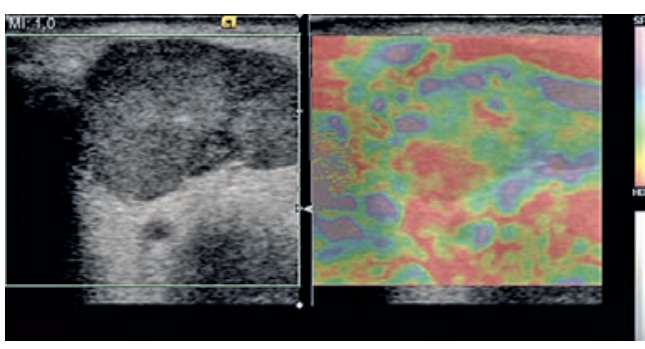

Fig 6. Pleomorphic adenoma. ARFI technique elastography. The tumor presents moderate stiffness in the centre and increased in the periphery.

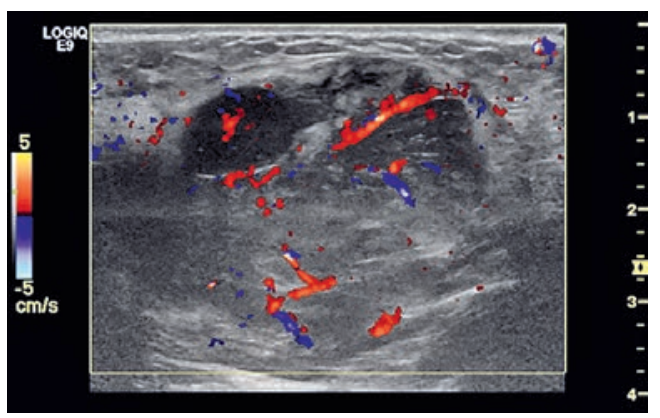

Fig 7. Pleomorphic adenoma. Color coded Doppler ultrasonography (CFM). The tumor vessels present a divergent, relatively uniform orientation.

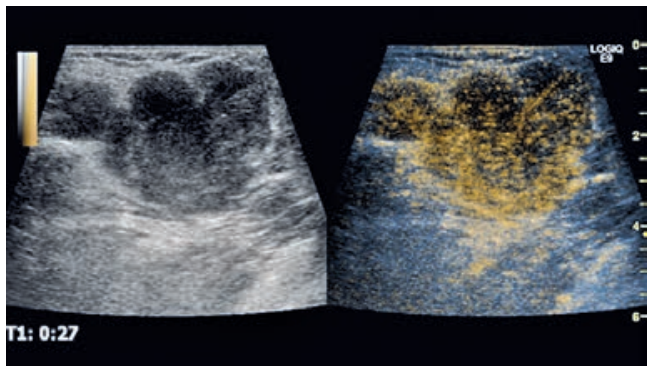

Fig 8. Parotid adenoma. Homogeneous enhancement pattern during the arterial phase $(27 \mathrm{sec})$.

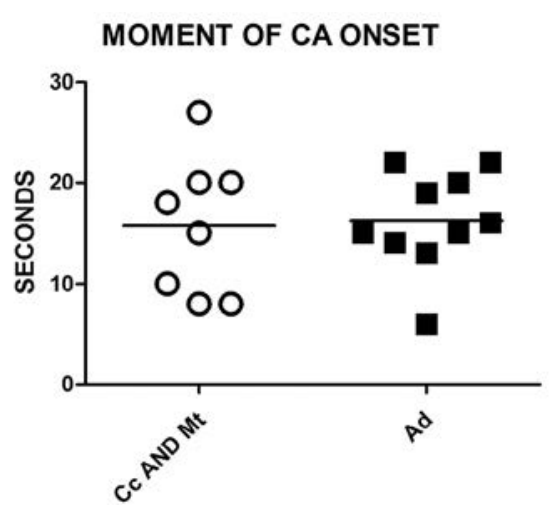

Fig 9. Times of CA onset dispersion in malignant (carcinomas and metastases) and benign (adenomas) parotid tumors. The line is drawn through the mean value. Cc-carcinomas, Mt-metastases, Ad-adenomas

\section{TTP}

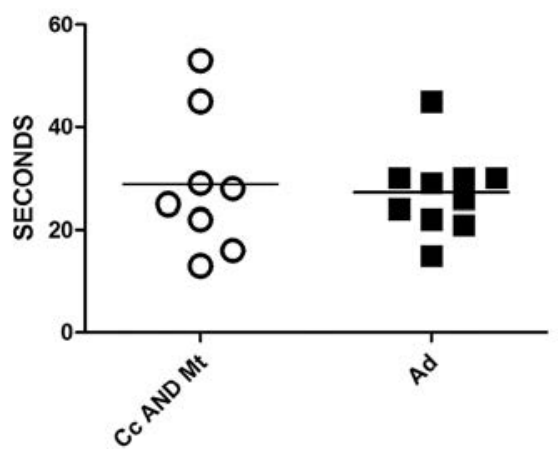

Fig 10. Dispersion of the time to peak (TTP) values in malignant (carcinomas and metastases) and benign (adenomas) parotid tumors. The line is drawn through the mean value. Cc-carcinomas, Mt-metastases, Ad- adenomas

PERSISTENCE OF THE CA

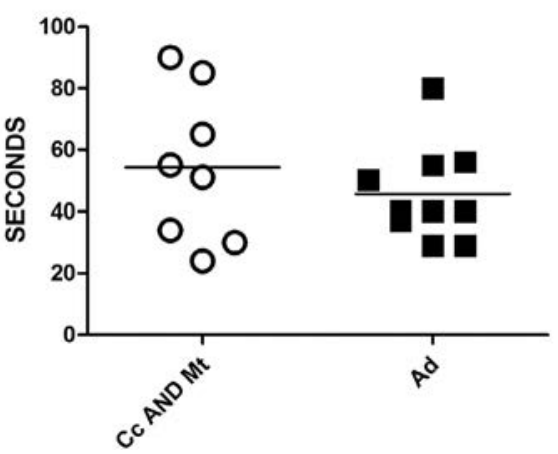

Fig 11. Dispersion of the times of overall CA enhancement of the tumor vascular bed in malignant (carcinomas and metastases) and benign (adenomas) parotid tumors. The line is drawn through the mean value. Cc-carcinomas, Mt-metastases, Ad- adenomas 
Table III. Ultrasonographic variables scaled by using decision trees

\begin{tabular}{|c|c|c|}
\hline Rank & Ultrasonographic variable & Significance \\
\hline 1 & Delineation & 1,0 \\
\hline 2 & Adenopathies & 1,0 \\
\hline 3 & Circulatory pattern & 0,997 \\
\hline 4 & Mobility & 0,991 \\
\hline 5 & Extension & 0,975 \\
\hline 6 & Enhancement pattern & 0,901 \\
\hline 7 & Vascular distribution & 0,869 \\
\hline 8 & Arterial resistance & 0,867 \\
\hline 9 & TTPs & 0,843 \\
\hline 10 & Stiffness & 0,809 \\
\hline 11 & Fluctuation & 0,692 \\
\hline 12 & Echogenicity & 0,692 \\
\hline 13 & DopplerSignal & 0,692 \\
\hline 14 & Vascular Type & 0,692 \\
\hline 15 & Elasticity & 0,65 \\
\hline 16 & CA persistance in ROIs & 0,583 \\
\hline 17 & CA onset & 0,236 \\
\hline 18 & VTVG Ratio & 0,215 \\
\hline 19 & Compressibility & 0,111 \\
\hline
\end{tabular}

TTP - Time to peak, CA - Contrast agent, ROI - Region of interest

to the normal parotid tissue (homogeneous/heterogeneous); pain upon palpation with the transducer; CEUS distribution in the tumor) (table III).

Decision trees have their own algorithm of selecting the relevant parameters and they identified three parameters: 1) tumor delineation 2) round hypoechoic cervical adenopathies, larger than $5 \mathrm{~mm}$, and 3) the onset time of the CA in the tumor assessed from the moment of the i.v. injection. The interpretation of the resulting decision trees was as it follows: if 1) the delineation of the tumor is clear $(0), 2)$ cervical adenopathies are present $(0)$ and 3 ) the onset time of the CA in the tumor is $<7$ seconds, then the diagnosis is benign (0). If the demarcation of the tumor is unclear (1) or capsular (2) then the diagnosis is malignant (1). If 1) the demarcation of the tumor is clear $(0)$, cervical adenopathies are absent (1), then the diagnosis is benign (0). If 1 ) the demarcation of the tumor is

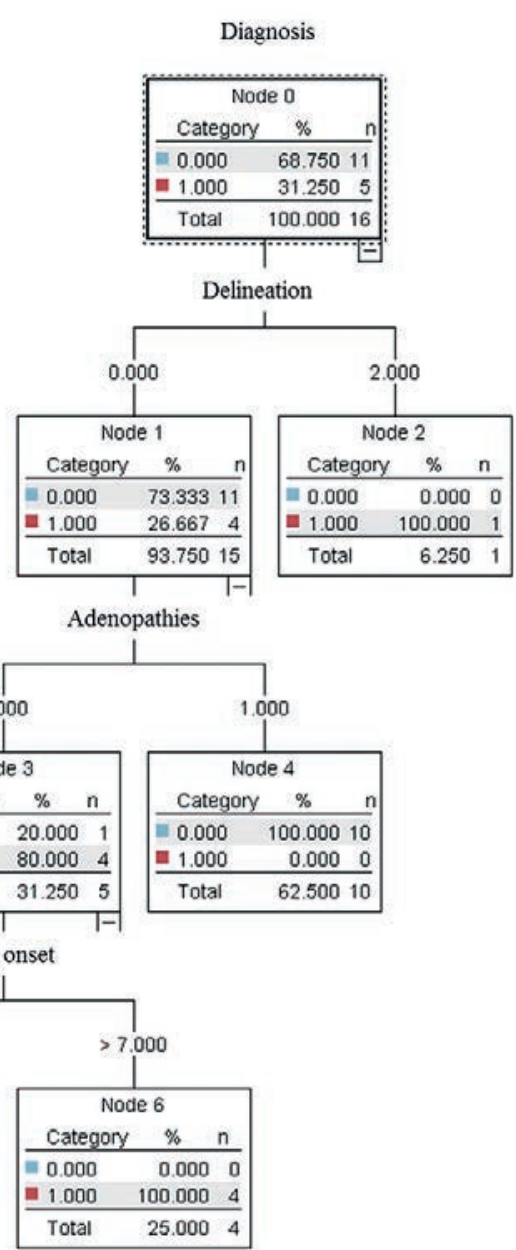

Fig 12. Decision trees and variables selected by the intelligent system.

clear (0), cervical adenopathies are present (0) and 3) the onset time of the CA in the tumor $>7$ seconds, then the diagnosis is malignant (1) (fig 12).

\section{Discussions}

Ultrasound is very accurate in detecting tumors as small as $5 \mathrm{~mm}$, based on its ability to discriminate between the normal tumoral parotid parenchyma. When in expert hands, this performance allows the identification of tumors in about $95 \%$ of cases! [10]. The difference between the generated echoes leads to the highlighting of a tumor wherever it may be, including in the parotid gland, if there aren't any gas or bone interferences, or if the lesion is not very far from the transducer [11]. The best and most proven performances of ultrasonography are in the detection and characterization of cysts and collec- 
tions [12]. Other indications for cervical ultrasound are: detection of lymph nodes and of diffuse salivary glands diseases [13].

The method is very accurate in detecting diffuse parotid diseases based on the changes of texture and on the increased size of the gland [14]. The US diagnosis of parotid disease is in direct relation with the occurrence of morphological changes, for which it expresses high sensitivity.

Unfortunately many of the parotid gland diseases progress up to a point showing identical structural changes [15]. One of the limits of ultrasonography is represented by its relatively low specificity.

A preselection of the cases characterized using ultrasonography may prove useful in formulating a prognosis. From this point of view a systematisation in cystic tumors (mostly benign) and solid masses (multiple etiologies) may be considered appropriate. A consequent more thorough characterization of the solid tumors in order to discriminate the ethiopathogenetic type is also important given the fact that the therapeutic conduct is very different $[2,16]$. In parotid adenomas the treatment is purely surgical, without representing an emergency. In parotid carcinoma or in metastases, the treatment of choice is urgent surgical removal followed by chemotherapy or radiation therapy, depending on the stage. Clearly staging is a key element of this therapy. The prompt characterization and discrimination between benign and malign tumors is thus of the essence.

The parotid ultrasonographic examination requires, in our opinion, a better definition, of an almost exhaustive nature, of the diagnostic criteria in solid tumors. The grey scale exploration is not sufficiently precise in achieving this goal [15].

Logically, these criteria should be anatomical, using a macroscopic anatomopathological description. They should be completed with mixed criteria, such as the anatomoclinical ones such as compressibility, stiffness, fluctuation and pain upon palpation. These features connect the clinical and ultrasound exams because of the dynamic or "real time" nature of the US information. The criteria must be completed with the hemodynamic information acquired using Doppler ultrasound and contrast enhanced ultrasonography. All these elements must use the latest ultrasound procedures in practice, including ultrasound elastography.

There are several types of approach in determining the diagnosis criteria for parotid masses [17]. The great variety of information provided by the ultrasound image explains all this. However no study has a sufficiently large group of cases to draw statistically significant conclusions [17].
In our opinion, morphological criteria represent the basis for the detection of solid tumors and their classification into benign or malignant. Unfortunately, ultrasound cannot generate an accurate diagnosis as far as the composition and the cellularity of a tumor is concerned. However, the spatial distribution of the tumor components, the identification of the areas that can be associated by analogy with areas of necrosis, of semi-fluid collection, of fibrosis and microcalcifications can be very useful in characterizing the tumor, all based on the ultrasound reflection of the collagen fibres and cellular elements of tissues [18]. The "assembling" pattern of these echoes must be compared with the anatomical sections of tumors. There are is a great variety of ultrasonographic features of these masses [19].

The US image should be regarded as a quite "rough" anatomic section, but also precise enough to guide the diagnosis. Therefore a very inhomogeneous echostructure and increased hypoechogenicity suggest a malignant tumor with underlying intratumoral nodular changes, perfusion abnormalities and areas of necrosis. Moderately inhomogeneous echostructure and moderate hypoechogenicity, closer to that of normal parenchyma, suggest an adenoma which is composed of fibrous septa and has a "mulberry-like" internal architecture. Imprecise delimitation suggests neoplastic infiltration, while clear delimitation suggests a benign, adenomatous tumor. Capsular continuity is directly correlated with the presence or absence of tumor invasion into adjacent structures. Cervical adenopathies when present and arranged in packets in the neighbourhood of the parotid gland represents a criterion for malignancy. Ultrasound semiology of adenopathies is already well known [20]. The method allows the discrimination of lymph nodes and their inclusion in the class of benign/malignant based on shape, delimitation, echogenicity, location of hilum and internal circulatory pattern, assessed by using CFM. Fluctuation and pain upon palpation of the mass are anatomoclinical criteria that confirm/rule out the inflammatory nature of the tumor.

A special attention should be given to tumor stiffness which can be assessed by using the elastographic techniques that were recently introduced in clinical practice [21]. There are many elastographic techniques, some of them based on tumor palpation (strain elastography), others based on the evaluation of the shear waves resulting from the US crossing through ROI (ARFI technique). The information obtained can be qualitative, by color coding, or quantitative, by measuring velocities expressed in meters/second. Our observation shows that malignant parotid tumors are rigid while the adenomas have a heterogeneous elastographic aspect. One explanation could be the very different composition of the tu- 
moral masses, with predominant nodular elements and microcalcifications in malignant tumors and fibrous textures and cell clumps in adenomas. To some extent, our study confirms the observations of other authors who have found a rather large variability of tumor elasticity which makes the method not sufficiently accurate when used alone [22]. But its integration within the broader context of procedures strengthens the diagnosis, mainly in respect to malignancy.

The circulatory model of the tumor can represent - as well - a diagnostic criterion [23]. This model is currently used in ultrasonography in the classification of liver masses (eg. the hepatocellular carcinoma is arterialised, with focally crowded vessels and chaotic spatial distribution), as well as renal, thyroid, breast, testicular masses etc. and to consolidate tumor diagnosis. Basically, this method allows the identification of tumor neovascularity and a definition of the inflammatory, benign or malignant vascular pattern [24]. An approach focused on tumor vascularity also requires the integration of existing methods. In this respect, ultrasonography provides information whose cumulative value can make important contributions to the diagnosis. For example, Doppler ultrasonography allows the study of the tumor feeding vessels and of the intratumoral hemodynamic features. Contrast ultrasound allows the assessment of blood dynamics through a circulatory bed, thus the two procedures are complementing each other.

Our study shows the need for the cumulative use of these criteria focused on the morphology and the hemodynamics of solid tumors of the parotid gland. Clearly, a „stratification" of these criteria must be realised based on their relevance. This stratification may take advantage of the decision trees support, as they have a very high accuracy, of up to $100 \%$, on present study data. Thus, the sum of the following criteria: inhomogeneous structure, increased hypoechogenicity, discontinuity of the parotid capsule and presence of adenopathies contribute greatly to the diagnosis of malignancy. Similar results were obtained by other authors using a comprehensive, multimodal approach. Thus, Knopf et al. in a recently published study shows a very high discrimination performance, up to $100 \%$, between benign parotid tumors and parotid carcinoma [25].

The originality of the study. Our study has a number of specific features that distinguish it from other original works. It is one of the few published studies that focuses on solid parotid tumors and takes into account all currently existing ultrasound procedures. Thus, it highlights, both individually and integratively, the value of the information this method can provide. It is also one of the first studies to demonstrate the applicability of i.v. CEUS in parotid pathology. CART decision trees were applied for the first time, their accuracy in supporting the diagnosis being up to $100 \%$.

Limitations of the study. The present study presents limitations related to the small number of cases within the study group. The results should therefore be interpreted with caution. It is obvious that a follow-up that includes a larger number of cases is needed. However it outlines a number of parameters that are clearly useful in prognosis. The use of analytical tools, such as specific elements of artificial intelligence and decision trees, partially exceed this impediment and make the results interesting. Another limitation of the study is the use of a wide range of equipment. This limitation has not affected the accuracy of the study, given the very modern technologies used in all cases.

\section{Conclusions}

The ultrasonographic exploration of solid parotid tumors requires a multimodal approach. The strong criteria for the diagnosis of malignancy are: unclear delineation of the gland, inhomogeneous structure, the presence of large cervical adenopathies, inhomogeneous vascularization, and uneven distribution of the circulatory bed when explored by CEUS. The criteria for the diagnosis of benign masses are: clear delineation, moderately inhomogeneous structure, lack of cervical adenopathies, homogeneous vascularity, and homogeneous distribution of the circulatory bed when explored by CEUS. In malignant tumors, the certainty changes are based on the existence of an advanced stage. The elastographic exploration is useful in the diagnosis of malignant tumors, but has a relative value in the diagnosis of adenomas. The CEUS exploration has a questionable value in the unilateral characterization of the nature of solid parotid tumors. Data mining approach, by using artificial intelligence, allows the development of intelligent diagnosis support systems, easy to use and interpret with an accuracy as high as $100 \%$.

Acknowledgements. We hereby thank the staff of the Ultrasonography Department of the $3^{\text {rd }}$ Medical Clinic for the support given in examining the patients.

Conflict of interest: none

\section{References}

1. Pinkston JA, Cole P. Incidence rates of salivary gland tumors: results from a population-based study. Otolaryngol Head Neck Surg 1999; 120: 834-840. 
2. Koch M, Zenk J, Iro H. Long-term results of morbidity after parotid gland surgery in benign disease. Laryngoscope 2010; 120: 724-730.

3. Lutz HT, Soldner R. Basic physics of ultrasound. In: Lutz $\mathrm{H}$, Buscarini $\mathrm{E}$ (eds). Manual of diagnostic ultrasound. $\left(2^{\text {nd }}\right.$ Ed). Vol. 1. World Health Organisation. Gutenberg Press Ltd, Malta 2011: 1-4.

4. Hertzberg BS, Kliewer MA, Bowie JD, et al. Physician training requirements in sonography: how many cases are needed for competence? AJR Am J Roentgenol 2000;174:1221-1227.

5. AIUM Practice Guideline for the Performance of an Ultrasound Examination of the Abdomen and/or Retroperitoneum. 2012, 1 - 12. http://www.aium.org/resources/guidelines/abd2011ominal.pdf

6. Cosgrove D. Achieving optimal diagnostic yield through the use of real-time contrast-enhanced ultrasonography (CEUS). Eur Radiol 2007; 17 [Suppl 6]: 71-72.

7. Wells PN, Liang HD. Medical ultrasound: imaging of soft tissue strain and elasticity. J R Soc Interface 2011; 8: 15211549.

8. Piscaglia F, Nolsøe C, Dietrich CF, et. al. The EFSUMB Guidelines and Recommendations on the Clinical Practice of Contrast Enhanced Ultrasound (CEUS): update 2011 on non-hepatic applications. Ultraschall Med 2012; 33: 33-59.

9. Nisbet R, Elder J, Miner G. Handbook of Statistical Analysis and Data Mining Applications. Academic Press, San Diego 2009.

10. Gritzmann N. Sonography of the salivary glands. AJR Am J Roentgenol 1989; 153: 161-166.

11. Dudea SM. Ultrasonography of the salivary glands: what's new? Med Ultrason 2010; 12: 173-174.

12. Stennert E, Wittekindt C, Klussmann JP, Guntinas-Lichius $\mathrm{O}$. New aspects in parotid gland surgery. Otolaryngol Pol 2004;58:109-114.

13. Dudea SM, Lenghel M, Botar-Jid C, Vasilescu D, Duma M. Ultrasonography of superficial lymph nodes: benign vs. malignant. Med Ultrason 2012;14:294-306.

14. Madani G, Beale T. Inflammatory conditions of the salivary glands. Semin Ultrasound CT MR 2006; 27: 440-451.
15. Bozzato A, Zenk J, Greess H, et al. Potential of ultrasound diagnosis for parotid tumors: analysis of qualitative and quantitative parameters. Otolaryngol Head Neck Surg 2007; 137: 642-646.

16. McGurk M, Thomas BL, Renehan AG. Extracapsular dissection for clinically benign parotid lumps: reduced morbidity without oncological compromise. Br J Cancer 2003; 89: 1610-1613.

17. Mansour N, Stock KF, Chaker A, Bas M, Knopf A. Evaluation of parotid gland lesions with standard ultrasound, color duplex sonography, sonoelastography, and acoustic radiation force impulse imaging - a pilot study. Ultraschall Med 2012; 33: 283-288.

18. Fields S, Dunn F. Letter: Correlation of echographic visualizability of tissue with biological composition and physiological state. J Acoust Soc Am 1973; 54: 809-812.

19. Fodor D, Pop S, Maniu A, Cosgaria M. Gray scale and Doppler ultrasonography of the benign tumors of parotid gland (pleomorphic adenoma and Warthin's tumor). Pictorial essay. Med Ultrason 2010; 12: 238-244.

20. Ahuja AT, Ying M, Ho SY, et al. Ultrasound of malignant cervical lymph nodes. Cancer Imaging 2008; 8: 48-56.

21. Bamber J, Cosgrove D, Dietrich CF, et al. EFSUMB guidelines and recommendations on the clinical use of ultrasound elastography. Part 1: Basic principles and technology. Ultraschall Med 2013; 34: 169-184.

22. Dumitriu D, Dudea S, Botar-Jid C, Baciut M, Baciut G. Real-time sonoelastography of major salivary gland tumors. AJR Am J Roentgenol 2011; 197: W924-W930.

23. Białek EJ, Jakubowski W, Karpińska G. Role of ultrasonography in diagnosis and differentiation of pleomorphic adenomas: work in progress. Arch Otolaryngol Head Neck Surg 2003; 129: 929-933.

24. Badea R, Socaciu M, Lupşor M, Moşteanu O, Pop T. Evaluating the liver tumors using three-dimensional ultrasonography. A pictorial essay. J Gastrointestin Liver Dis 2007; 16: 85-92.

25. Knopf A, Mansour N, Chaker A, Bas M, Stock K. Multimodal ultrasonographic characterisation of parotid gland lesions--a pilot study. Eur J Radiol 2012; 81: 3300-3305. 